PHYSICAL REVIEW D 96, 126008 (2017)

\title{
Unitarity restoring graviton radiation in the collapse regime of gravitational scattering
}

\author{
Marcello Ciafaloni* \\ Dipartimento di Fisica, Università di Firenze and INFN Firenze Via Sansone 1, \\ 50019 Sesto Fiorentino, Italy \\ Dimitri Colferai ${ }^{\dagger}$ \\ Dipartimento di Fisica, Università di Firenze and INFN Firenze Via Sansone 1, \\ 50019 Sesto Fiorentino, Italy
}

(Received 17 October 2017; published 14 December 2017)

\begin{abstract}
We investigate graviton radiation in gravitational scattering at small impact parameters $b<R \equiv 2 G \sqrt{s}$ and extreme energies $s \gg M_{P}^{2}$, a regime in which classical collapse is thought to occur, and thus radiation may be suppressed also. Here however, by analyzing the soft-based representation of radiation recently proposed in the semiclassical Amati-Ciafaloni-Veneziano framework, we argue that gravitons can be efficiently produced in the untrapped region $|\boldsymbol{x}| \gtrsim R>b$, so as to suggest a possible completion of the unitarity sum. In fact, such energy radiation at large distances turns out to compensate and to gradually reduce to nothing the amount of energy $E^{\prime}$ being trapped at small- $b$ 's, by thus avoiding the quantum tunneling suppression of the elastic scattering and suggesting a unitary evolution. We finally look at the coherent radiation sample so obtained and we find that, by energy conservation, it develops an exponential frequency damping corresponding to a "quasitemperature" of order $\hbar / R$, which is naturally related to a Hawking radiation and is suggestive of a black-hole signal at quantum level.
\end{abstract}

DOI: $10.1103 /$ PhysRevD.96.126008

\section{INTRODUCTION}

The thought experiment of point particle gravitational scattering at trans-Planckian energies has been investigated, since the eighties [1-7], as a probe of quantum-gravity theories, mostly in connection with the problem of a possible loss of quantum coherence in a process leading classically to gravitational collapse and perhaps black-hole formation. In an $S$-matrix framework such a loss would be associated with the breakdown of unitarity at sufficiently small impact parameters.

In the scattering regime of large energies $(\sqrt{s} \equiv 2 E \gg$ $M_{P}$ ) but small deflection angles (i.e., in a regime far away from that of collapse), several authors proposed [1-5], on various grounds, an approximate semiclassical description, whose $S$-matrix exponentiates an eikonal function of order $\alpha_{G} \equiv G s / \hbar \gg 1, G$ being the Newton constant, which corresponds to the $s$-channel iteration of the spin-2 graviton exchange at impact parameters $b$ much larger than the gravitational radius $R \equiv 2 G \sqrt{s}$. Such description implies that the large momentum transfer built up at fixed Einstein deflection angle $\Theta_{E} \equiv 2 R / b$ and trans-Planckian energies is due to a large number $\langle n\rangle \sim \alpha_{G} \gg 1$ of single hits with very small scattering angle $\theta_{m} \sim \hbar / b E$.

Starting from that leading eikonal approximation, the strategy followed in $[6,7]$ consisted in a systematic study of

*ciafaloni@fi.infn.it

†colferai@fi.infn.it subleading corrections to the eikonal phase, scattering angle, and time delays [8-10] in terms of the expansion parameter $R^{2} / b^{2}$ (and possibly $l_{s}^{2} / b^{2}$ if working within string theory at scale $l_{s}$ ). These corrections can be resummed, in principle, by solving a classical field theory and one can thus study the critical region $b \sim R$ where gravitational collapse is expected.

That Amati-Ciafaloni-Veneziano (ACV) program (the "ACV resummation") was carried out, neglecting string corrections and after a drastic axisymmetric truncation (the "reduced-action model") of the classical field theory due to Lipatov [11], in Ref. [12] (see also [13-15]). It was noted there that below some critical impact parameter value $b_{c} \sim R$, the elastic $S$-matrix-evaluated by taking UV-safe, but possibly complex, solutions of the field equationsshows a unitarity deficit.

Indeed, the resummed eikonal of the axisymmetric reduced-action model has the simple form $[12]^{1}$

$$
\begin{aligned}
\delta(\boldsymbol{b}, s) & =\alpha_{G}\left[\log \frac{L}{R}+\Delta(\boldsymbol{b})\right], \\
\Delta(\boldsymbol{b}) & \equiv-\mathrm{a} \tanh \left(t_{b}\right)+\frac{1}{2}-\frac{1}{2 t_{b}},
\end{aligned}
$$

\footnotetext{
${ }^{1}$ Boldface variables $\boldsymbol{b}, \boldsymbol{q}$ etc. denote two-dimensional Euclidean transverse components, while three-dimensional spatial vectors are marked by an arrowlike $\vec{q}$.
} 
where $t_{b}(b / R)$ is a properly chosen solution of the criticality equation

$$
t_{b}\left(1-t_{b}^{2}\right)=\frac{R^{2}}{b^{2}}
$$

and $L$ is an IR cutoff, factorized in the elastic $S$-matrix expression

$$
S_{\mathrm{el}}(\boldsymbol{b}, s)=\mathrm{e}^{2 \mathrm{i} \delta(\boldsymbol{b}, s)}=\mathrm{e}^{2 \mathrm{i} \alpha_{G} \log \frac{L}{R}} \mathrm{e}^{2 \mathrm{i} \alpha_{G} \Delta(\boldsymbol{b})} .
$$

We notice that the leading contribution $\alpha_{G} \log (R / b)$ is corrected in $\Delta(\boldsymbol{b})$ by higher order terms, corresponding to irreducible diagrams [7] of the relevant effective action, which are thereby resummed. Furthermore, the criticality equation (1.2) identifies a branch-cut singularity of the series at $b^{2}=b_{c}^{2} \equiv(3 \sqrt{3} / 2) R^{2}$, below which the eikonal function acquires a (positive) imaginary part. As a consequence, for $b>b_{c}$ the perturbative solution $\left(t_{b} \rightarrow 1\right.$ for $b \gg R$ ) keeps the eikonal a pure phase. But for $b<b_{c}$, by choosing the appropriate [12] $t_{b}$ branch, the elastic scattering amplitude acquires the suppression factor

$$
\left|S_{\mathrm{el}}(\boldsymbol{b}, s)\right| \simeq \begin{cases}\exp \left[-\frac{2 \sqrt{2}}{3} \alpha_{G}\left(1-\frac{b^{2}}{b_{c}^{2}}\right)^{3 / 2}\right] & \left(b_{c}-b \ll R\right) \\ \exp \left[-\alpha_{G}\left(\pi-\frac{3 \sqrt{3}}{2}\left(\frac{b}{R}\right)^{2 / 3}\right)\right] & (b \ll R)\end{cases}
$$

which eventually forbids the direct observation of the trapped energy $2 E$ in the elastic channel.

That conclusion was confirmed, at quantum level, by the interpretation of the above (complex) restricted solutions as due to tunneling through a barrier in metric space [16], which is classically forbidden. Therefore, by the apparent lack of sufficiently large inelastic contributions to unitarity, the above results seem to imply that the lost information could possibly be recovered only through use of UVsensitive solutions which, by definition, cannot be studied by the effective action approach.

On the other hand, the more recent investigation of graviton radiation associated to trans-Planckian scattering at both classical [17] and quantum level [18-20] has brought up in various approaches some interesting new features which suggest a solution for the unitarity problem at a simpler and more conservative level. First of all, the emission energies $\hbar \omega$ we are interested in are mostly of order $\hbar / R \sim$ $M_{P}^{2} / E \ll M_{P}$ and are thus soft at trans-Planckian energies, by calling attention to soft physics (as emphasized e.g. in $[21,22])$ besides high-energy scattering. That poses a nontrivial consistency requirement on our graviton radiation amplitude, because it should agree with both the emission implied by the Weinberg current [23] in the fragmentation region and with that required by the high-energy Lipatov current [11] in the central region. Starting from that observation, a unified approach to both regimes has been proposed in $[18,19]$, leading to the so-called "soft-based representation" of graviton emission to be summarized shortly in Sec. II. Furthermore, the latter has been tentatively extended [20] to the eikonal resummation of the reducedaction model [12] just mentioned, by making it possible to investigate the small impact parameter region $b \lesssim b_{c}$, within the drastic assumptions implied by that model.

Given the new context emphasized above, the purpose of the present paper is to reexamine the unitarity issue at small impact parameters by taking advantage of the soft-based representation of graviton radiation, and to show how it is possibly solved, by emphasizing the role of multigraviton states which turn out to act as a key to weaken and, eventually, to pass the metric barrier.

Before presenting our arguments, we need to make two premises. First, the soft-based representation has already been used for $b>b_{c}[18,19]$, where unitarity is not at stake, by emphasizing the role of the gravitational radius $R$ in the radiation process. Indeed, by combining the relatively small emitted energy $(\hbar \omega / E \ll 1)$ with the large number $\langle n\rangle \sim \alpha_{G}=E R / \hbar$ of single hits in eikonal scattering, the variable $\omega R$ emerges and identifies the main features of the energy emission distribution. The outcome is an operator $S$-matrix combining the resummed eikonal and radiation in a (unitary) coherent state

$$
\begin{aligned}
\hat{S}= & \mathrm{e}^{2 \mathrm{i} \delta(\boldsymbol{b}, s)} \exp \left\{\int \frac { \mathrm { d } ^ { 3 } q } { \hbar ^ { 3 } \sqrt { 2 \omega } } 2 \mathrm { i } \left[\sum_{\lambda} \mathfrak{M}_{\lambda}(\boldsymbol{b}, \vec{q}) a_{\lambda}^{\dagger}(\vec{q})\right.\right. \\
& + \text { H.c. }]\},
\end{aligned}
$$

where the probability amplitude $\mathfrak{M}_{\lambda}(\boldsymbol{b}, \vec{q})$ for emitting a graviton with momentum $\vec{q}$ and helicity $\lambda$ is explicitly given and briefly constructed in Sec. II, while $a_{\lambda}^{\dagger}(\vec{q})$ is the corresponding creation operator. Unitarity here is supposed to follow from appropriate and factorized virtual corrections [Eq. (3.4)], which are argued for in Secs. II and III, but are not really derived from some underlying quantumgravity theory. For that reason we prefer to talk of "unitarization procedure" rather than "unitarity proof" in passing from the tree-level amplitude $\mathfrak{M}$ to the final unitary coherent state (1.5), with its factorized emission structure.

Secondly, we have shown [20] that such an (approximate) independent-particle picture can be extended to the approach-to-collapse regime $b \rightarrow b_{c}^{+}$of enhanced radiation by incorporating energy-conservation constraints in the unitarization procedure. The latter cause the emergence of a novel, exponential frequency damping whose coefficient $\bar{\tau} R$ defines what we call a quasitemperature $T \equiv \hbar /(\bar{\tau} R)$ which is naturally related to the Hawking temperature $[24,25]$. The main difference, though — and the motivation for its unconventional name-is that $\bar{\tau}$ is supposed to keep quantum coherence, and is not due to a statistical averaging. Rather, the lack of sizeable correlations in such a result is due to the soft-graviton dynamics we started with, which led to the unified form of the $b \rightarrow b_{c}^{+}$emission amplitudes. 
Finally, the basic question comes back: what about the collapse regime of $b<b_{c}$, in which the energy $2 E$ appears to be "trapped" because elastic unitarity is exponentially violated by the suppression factors (1.4) without apparent contributions in the most naive radiation models $[8,16,26,27]$ ? In other words, is unsuppressed radiation predictable for $b<b_{c}$ in our present soft-based representation? That is precisely the question that we address in this paper. We argue that during eikonal scattering, the soft-radiation process has indeed the ability to reduce the amount of trapped energy crossing the barrier, and thus to weaken and, eventually, to eliminate (Sec. III) the suppression factor (1.4). As a consequence (Secs. IV and V), our coherent radiation sample can efficiently contribute to the unitarity sum, and still may have a normal quasitemperature of order $\hbar / R$. For that reason, it is a good candidate for the generalized unitarization procedure that we describe, as discussed in Sec. VI.

\section{UNIFIED SOFT-BASED REPRESENTATION OF SINGLE-EMISSION AMPLITUDE}

We start, in the ACV framework, from the (irreducible) resummed eikonal $\alpha_{G} \Delta(\boldsymbol{b})$ in Eq. (1.1), whose Fourier transform defines a "potential" $\tilde{\Delta}(\boldsymbol{Q})$ in transverse space. In the soft limit and in the fragmentation region, the emission amplitude is then given by the external-line insertion formula, which factorizes in $Q$-space as follows $(\hbar=1$ from now on):

$$
\begin{aligned}
\mathcal{M}_{\lambda}^{\text {soft }}(\boldsymbol{b}, E, \boldsymbol{q}, \omega) & =\sqrt{\alpha_{G}} \frac{R}{\pi} \int \frac{\mathrm{d}^{2} \boldsymbol{Q}}{2 \pi} \tilde{\Delta}(\boldsymbol{Q}) \mathrm{e}^{\mathrm{i} \boldsymbol{Q} \cdot \boldsymbol{b}} \\
& \times \frac{1}{2}\left[\frac{E}{\omega}\left(\mathrm{e}^{-\mathrm{i} \lambda\left(\phi_{\left.q-\frac{\omega}{E} Q^{-} \phi_{q}\right)}\right.}-1\right)\right],
\end{aligned}
$$

where $\boldsymbol{q}$ is the transverse momentum of the emitted graviton, $\phi_{q}$ is its azimuth in the transverse plane, $\lambda=$ \pm 2 is its helicity, and the factor in square brackets comes from the explicit computation of the Weinberg current on helicity states [18].

It was shown in [19] that a similar formula is able to describe graviton emissions in the central region-where the Lipatov current [28] should be used in the high-energy limit—by just performing a simple subtraction of the same expression at scale $E \rightarrow \omega$. The unified single-emission amplitude thus reads

$$
\begin{aligned}
\mathcal{M}_{\lambda}^{[1]}(\boldsymbol{b}, E, \boldsymbol{q})= & -\sqrt{\alpha_{G}} \frac{R}{\pi} \mathrm{e}^{\mathrm{i} \lambda \phi_{q}} \int \frac{\mathrm{d}^{2} \boldsymbol{x}}{2 \pi|\boldsymbol{x}|^{2} \mathrm{e}^{\mathrm{i} \lambda \phi_{x}}} \mathrm{e}^{\mathrm{i} q \cdot \boldsymbol{x}} \\
& \times\left[\frac{E}{\omega}\left(\Delta\left(\boldsymbol{b}-\frac{\omega}{E} \boldsymbol{x}\right)-\Delta(\boldsymbol{b})\right)\right. \\
& -(\Delta(\boldsymbol{b}-\boldsymbol{x})-\Delta(\boldsymbol{b}))]
\end{aligned}
$$

where we have exchanged the $\boldsymbol{Q}$ integration with an $\boldsymbol{x}$ integration that provides a convenient representation of phase transfers (e.g. for $\lambda=-2$ ),

$$
\mathrm{e}^{2 \mathrm{i} \phi \boldsymbol{\theta}}-\mathrm{e}^{2 \mathrm{i} \phi_{\boldsymbol{\theta}^{\prime}}}=-2 \int \frac{\mathrm{d}^{2} \boldsymbol{x}}{2 \pi x^{* 2}}\left(\mathrm{e}^{\mathrm{i} A x \cdot \boldsymbol{\theta}}-\mathrm{e}^{\mathrm{i} A x \cdot \boldsymbol{\theta}^{\prime}}\right),
$$

where $A$ is a nonvanishing real-valued constant and $x \equiv x_{1}+\mathrm{i} x_{2}, x^{*} \equiv x_{1}-\mathrm{i} x_{2}$ is the complex notation for the transverse vector $\boldsymbol{x}$.

We notice that Eq. (2.2) is directly expressed in terms of the eikonal function $\alpha_{G} \Delta(\boldsymbol{b})$ of Eq. (1.1), which occurs in the so-called modulating function

$$
\begin{aligned}
\Phi(\omega, \boldsymbol{x}) & \equiv \frac{E}{\omega}\left[\Delta\left(\boldsymbol{b}-\frac{\omega}{E} \boldsymbol{x}\right)-\Delta(\boldsymbol{b})\right]-[\Delta(\boldsymbol{b}-\boldsymbol{x})-\Delta(\boldsymbol{b})] \\
& \equiv \Phi_{A}(\boldsymbol{x})-\Phi_{B}(\boldsymbol{x}),
\end{aligned}
$$

where the first (second) term is in correspondence with external (internal) insertions. Thus, the single-exchange amplitude (2.2) measures the Fourier transform of the "soft field"

$$
h_{s}^{(\lambda)}(\omega, \boldsymbol{x})=-\frac{\Phi(\omega, \boldsymbol{x})}{\pi^{2}|\boldsymbol{x}|^{2} \mathrm{e}^{\mathrm{i} \lambda \phi_{x}},}
$$

which plays an important role in the expression of the ACV metric also [19].

For the purpose of orientation, we note that in the $\omega \rightarrow 0$ limit, (2.4) becomes

$$
\Phi(0, \boldsymbol{x})=-\Delta^{\prime}(b) \boldsymbol{x} \cdot \hat{\boldsymbol{b}}+\Delta(\boldsymbol{b})-\Delta(\boldsymbol{b}-\boldsymbol{x})
$$

an expression in which the term linear in $\boldsymbol{x} \cdot \hat{\boldsymbol{b}}$, proportional to the classical scattering angle ${ }^{2}$ of order $\Theta_{E} \equiv 2 R / b$, dominates in the fragmentation region thus reproducing the Weinberg results [19].

The full graviton radiation amplitude is now obtained by superimposing the single-exchange amplitudes (2.2) over all the $n$ rungs of the eikonal diagrams (Fig. 1). The contribution of the $j$ th rung can be written as

$$
\begin{aligned}
\mathcal{M}_{\lambda}^{[n, j]}(\boldsymbol{b}, E, \boldsymbol{q})= & \mathrm{e}^{\mathrm{i} \lambda \phi_{\theta}} \sqrt{\alpha_{G}} \frac{R}{2} \frac{\left(2 \mathrm{i} \alpha_{G}\right)^{n-1}}{n !} \\
& \times \int \mathrm{d}^{2} \boldsymbol{x} \mathrm{e}^{\mathrm{i} \boldsymbol{q} \cdot \boldsymbol{x}}\left[\Delta\left(\boldsymbol{b}-\frac{\omega}{E} \boldsymbol{x}\right)\right]^{j-1} h_{s}^{(\lambda)}(\omega, \boldsymbol{x}) \\
& \times[(E-\omega) \Delta(\boldsymbol{b})+\omega \Delta(\boldsymbol{b}-\boldsymbol{x})]^{n-j},
\end{aligned}
$$

where we notice two important effects. First, the $j$ th incidence angle is rotated with respect to the $z$-axis, by

\footnotetext{
${ }^{2}$ The estimate $\Theta_{\mathrm{cl}}=-2 R \Delta^{\prime}(\boldsymbol{b})$ comes from a saddle point evaluation of the elastic scattering amplitude as a Fourier transform of Eq. (1.3).
} 


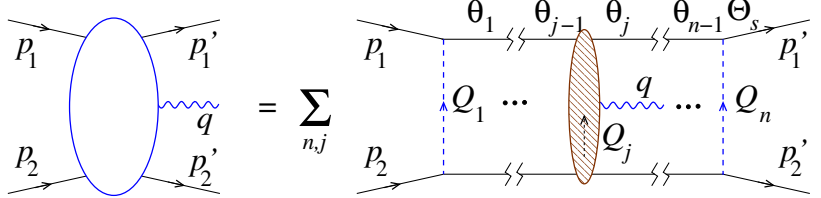

FIG. 1. Graviton emission from the eikonal ladder. The $n$-rung diagram with the emission from the $j$ th exchange is denoted by $\mathcal{M}^{[n, j]}$ in the text.

translating the $\boldsymbol{\theta}$-dependence by the quantity $\Theta_{j}=$ $\left(\boldsymbol{Q}_{1}+\cdots+\boldsymbol{Q}_{j-1}\right) / E$. That produces in turn, after Fourier transform, the shift $-(\omega / E) \boldsymbol{x}$ in the impact parameter of the elastic amplitudes before emission.

Secondly, after emission we have the rescattering effects: the energetic particle acquires the recoil energy $E-\omega$, and the emitted graviton at position $\boldsymbol{x}$ rescatters with energy $\omega$ and relative impact parameter $\boldsymbol{b}-\boldsymbol{x}$.

Finally, the summation over $j$ of the contributions (2.7) is performed by the formula

$\sum_{n=1}^{\infty} \frac{1}{n !} \sum_{j=1}^{n} A^{j-1} B^{n-j}=\sum_{n=0}^{\infty} \frac{1}{n !} \frac{A^{n}-B^{n}}{A-B}=\frac{\mathrm{e}^{A}-\mathrm{e}^{B}}{A-B}$,

where

$$
\begin{aligned}
& A \equiv 2 \mathrm{i} \alpha_{G}\left[\Delta(\boldsymbol{b})+\frac{\omega}{E} \Phi_{A}(\boldsymbol{x})\right], \\
& B \equiv 2 \mathrm{i} \alpha_{G}\left[\Delta(\boldsymbol{b})+\frac{\omega}{E} \Phi_{B}(\boldsymbol{x})\right]
\end{aligned}
$$

are given in terms of the $\Phi_{A, B}$ of Eq. (2.4). We thus realize that the factor $\Phi(\boldsymbol{x})=\Phi_{A}-\Phi_{B}$ in the soft field $h_{s}^{(\lambda)}$ cancels out with the $A-B$ denominator of the summation (2.8). That cancellation is somewhat surprising. Somehow, the identity used in Eq. (2.2)

$$
\mathcal{M}_{\text {matched }}=\left.\operatorname{soft}\right|_{E}-\left.\operatorname{soft}\right|_{\omega} \text {, }
$$

that was interpreted as a decomposition of external plus internal insertions in the soft language, acquires now the interpretation of (A) "incidence-changing" plus (B) rescattering terms in the high-energy language.

The final result can thus be written as

$$
\begin{aligned}
\mathcal{M}_{\lambda}(\boldsymbol{b}, E, \boldsymbol{q})= & \mathrm{e}^{2 \mathrm{i} \alpha_{G} \Delta(\boldsymbol{b})} \mathfrak{M}_{\lambda}(\boldsymbol{b}, \omega, \boldsymbol{q}) \\
\mathfrak{M}_{\lambda}(\boldsymbol{b}, \omega, \boldsymbol{q})= & \sqrt{\alpha_{G}} \frac{R}{\pi} \mathrm{e}^{\mathrm{i} \lambda \phi_{q}} \int \frac{\mathrm{d}^{2} \boldsymbol{x}}{2 \pi|\boldsymbol{x}|^{2} \mathrm{e}^{\mathrm{i} \lambda \phi_{x}}} \mathrm{e}^{\mathrm{i} \boldsymbol{q} \cdot \boldsymbol{x}} \mathrm{e}^{2 \mathrm{i} \omega R \Phi_{A}(\boldsymbol{x})} \\
& \times \frac{\mathrm{e}^{-2 \mathrm{i} \omega R \Phi(\boldsymbol{x})}-1}{2 \mathrm{i} \omega R}
\end{aligned}
$$

where $\mathfrak{M}_{\lambda}$ is the probability amplitude occurring in the unitary coherent state (1.5).

\section{MULTIGRAVITON EMISSION AND UNSUPPRESSED RADIATION IN THE COLLAPSE REGIME}

As we have just seen, the Ciafaloni-Colferai (CC) [20] method for incorporating the resummed eikonal $\alpha_{G} \Delta(\boldsymbol{b})$ of Eq. (1.1) in the radiation process is best illustrated (Fig. 2) by the one-graviton emission amplitude

$$
\begin{aligned}
\mathcal{M}_{2 \rightarrow 3}= & \int \frac{\mathrm{d}^{2} \boldsymbol{x}}{(2 \pi)^{2}} \frac{\sqrt{\alpha_{G}}}{x^{* 2}} \frac{\mathrm{e}^{\mathrm{i} \boldsymbol{q} \cdot \boldsymbol{x}}}{\mathrm{i} \omega} \\
& \times\left[\mathrm{e}^{2 \mathrm{i}(E-\omega) R \Delta(\boldsymbol{b})+2 \mathrm{i} \omega R \Delta(\boldsymbol{b}-\boldsymbol{x})}-\mathrm{e}^{2 \mathrm{i} \alpha_{G} \Delta\left(\boldsymbol{b}-\frac{\omega}{E} \boldsymbol{x}\right)}\right]
\end{aligned}
$$

which contains (i) the rescattering term with its typical $(E-\omega)$ recoil energy (which is here listed as the first term) and (ii) the incidence-changing term with its $\omega$-dependent shift (second term). From Eq. (3.1), by dividing out the $2 \rightarrow 2 S$-matrix $\exp \left[2 \mathrm{i} \alpha_{G} \Delta(\boldsymbol{b})\right]$, we obtain the singleemission probability amplitude of Eq. (2.11),

$$
\begin{aligned}
\mathfrak{M}_{\lambda}(\boldsymbol{b}, \omega, \boldsymbol{q}) \simeq & \int \frac{\mathrm{d}^{2} \boldsymbol{x}}{(2 \pi)^{2}} \frac{\sqrt{\alpha_{G}}}{|x|^{2} \mathrm{e}^{\mathrm{i} \lambda \phi_{\boldsymbol{x}}}} \frac{\mathrm{e}^{\mathrm{i} \boldsymbol{q} \cdot \boldsymbol{x}}}{\mathrm{i} \omega} \\
& \times\left\{\mathrm{e}^{-2 \mathrm{i} \omega R[\Delta(\boldsymbol{b})-\Delta(\boldsymbol{b}-\boldsymbol{x})]}-1\right. \\
& \left.+1-\mathrm{e}^{2 \mathrm{i} \alpha_{G}\left[\Delta\left(\boldsymbol{b}-\frac{\omega}{E} \boldsymbol{x}\right)-\Delta(\boldsymbol{b})\right]}\right\},
\end{aligned}
$$

where in curly brackets we have singled out the rescattering and incidence-changing terms.

Multiple emission from the eikonal ladder is then treated by summing up the various rungs' contributions by generalizing Fig. 1. That procedure, carried out in Refs. [19,20], shows that, in the soft region $\omega_{i} \ll E$, real emission factorization holds in the form

$$
\begin{aligned}
\mathcal{M}_{2 \rightarrow 2+N} \simeq & \mathrm{e}^{2 \mathrm{i} \alpha_{G} \Delta(\boldsymbol{b})} \prod_{i=1}^{N} \mathfrak{M}_{\lambda_{i}}\left(\boldsymbol{b}, \omega_{i}, \boldsymbol{q}_{i}\right) \\
& \times\left[1+\mathcal{O}\left(\frac{\omega_{j}^{2}}{E^{2}}\right)\right] .
\end{aligned}
$$

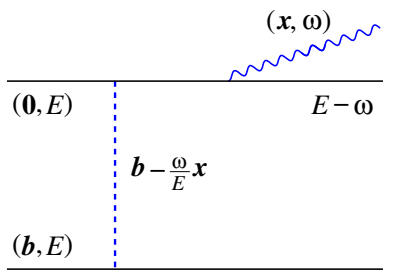

(a)

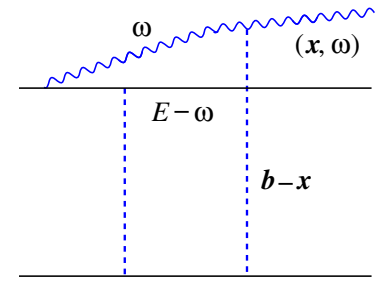

(b)
FIG. 2. Radiation diagram of the soft-based representation: (a) External line insertion shifts the impact parameter $\boldsymbol{b}-\frac{\omega}{E} \boldsymbol{x}$ and rotates the incidence angle. (b) Internal line insertion scatters at recoil energy $E-\omega$ and rescatters at position $\boldsymbol{b}-\boldsymbol{x}$ and energy $\omega$. 
In the case $b>b_{c}, \Delta(\boldsymbol{b})$ is real and the factorization (3.3) can be extended to virtual corrections by addition of a factor $\sqrt{\mathcal{P}_{0}}$, the no-emission amplitude, where

$$
\mathcal{P}_{0}=\exp \left\{-2 \int \frac{\mathrm{d}^{3} q}{\omega} \sum_{\lambda}\left|\mathfrak{M}_{\lambda}\right|^{2}\right\}
$$

is the no-emission probability. Equation (3.3), with the correction factor (3.4) is then equivalent to using the unitary $S$-matrix parametrization

$$
\begin{gathered}
S=\mathrm{e}^{2 \mathrm{i} \delta} \exp \left\{\int \frac{\mathrm{d}^{3} q}{\sqrt{2 \omega}} 2 \mathrm{i} \sum_{\lambda}\left[\mathfrak{M}_{\lambda} a_{\lambda}^{\dagger}(q)+\mathfrak{M}_{\lambda}^{*} a_{\lambda}(q)\right]\right\} \\
\omega P(\omega)=2 \sum_{\lambda} \int \mathrm{d}^{2} \boldsymbol{q}\left|\mathfrak{M}_{\lambda}\right|^{2}
\end{gathered}
$$

corresponding to the linear coherent-state operator (1.5) and to its emission density.

In [20] we noticed that the independent-particle picture just outlined is not fully consistent when scattering angle and coupling enter the collapse region and radiation should be corrected for energy-conservation effects. The latter can be taken into account by introducing, besides $\sqrt{\mathcal{P}_{0}}$, an amplitude renormalization factor $1 / \sqrt{\mathcal{N}(E)}$, where $\mathcal{N}(E)$ is dependent on the available energy $E$ and is determined by unitarity, by including the kinematical constraints event by event. The outcome is then the occurrence of the quasitemperature factor $\mathrm{e}^{-\omega / T}$ in inclusive distributions [20], which allows the correspondence of our coherent radiation sample with a Hawking radiation.

The case $b<b_{c}$ is deeply different, however. In fact, in such a case $\Delta(\boldsymbol{b})$ acquires an imaginary part which is of size $\mathrm{i} \pi / 2$ in the $b \ll R$ limit of deep collapse and has the interpretation of tunneling through a barrier [16]. As a consequence, the elastic amplitude in the ansatz (3.3) is exponentially suppressed like $\mathrm{e}^{-\pi \alpha_{G}}=\mathrm{e}^{-\pi E R}$ (for $E=\sqrt{s} / 2$ ), and thus the combined use of Eq. (3.3) and (3.4) cannot possibly correspond to a unitary parametrization of type (3.5). How does one reach unitarity then?

Our purpose here is to single out those emission processes which, on the basis of (3.1) and (3.2), are not suppressed and can possibly lead to unitarity recovery. We start noticing that, even at small impact parameters $b \ll R$, the emission amplitude (3.2) stays unsuppressed in (3.3) if the graviton is emitted at sufficiently large $|\boldsymbol{x}|$, outside the trapped region, so that the corresponding eikonal is real valued. For the rescattering terms [with $\Delta(\boldsymbol{b}-\boldsymbol{x})]$ such an "exit" occurs already at $|\boldsymbol{x}|>R \gg b$, while for the incidence-changing terms [with $\Delta\left(\boldsymbol{b}-\frac{\omega}{E} \boldsymbol{x}\right)$ ] we have to require that $|\boldsymbol{x}|>(E / \omega) R$ be much larger, in which case the $\boldsymbol{x}$-integration is suppressed by a phase space factor $\omega^{2} / E^{2}$ in the interesting soft region $\omega R \ll \alpha_{G}$.

Therefore, for $b \ll R$, the rescattering terms appear to provide the best visible radiation window and are investigated first at multigraviton level in the following. Indeed, according to Sec. II of [20], the multigraviton factorization (3.3) appears to hold exactly in the rescattering case-if incidence-changing terms are turned off-because the $\omega_{j} R$-dependence is simply additive. Furthermore, the probability amplitude $\mathfrak{M}$ of the rescattering term in (3.2) is increasing like $\exp (\pi \omega R)^{3}$ within the kinematical bounds and thus yields a strong radiation enhancement. The physical reason for such an increase is just energy conservation, because the recoil energy $(E-\omega)$ signals the corresponding suppression decrease.

As a consequence, by collecting all exponential terms and discretizing the phase space in energy intervals $\left[\omega_{j}, \omega_{j}+\Delta \omega_{j}\right]$, the independent particles' distribution reads, approximately, in each one of the forward and backward "jets,"

$$
\begin{aligned}
\mathrm{d} \mathcal{P}\left(\left\{\omega_{j}, N_{j}\right\}\right)= & \mathcal{P}_{\mathrm{el}}(E) \Theta\left(E-\sum_{j} \omega_{j} N_{j}\right) \\
& \times \prod_{j} \frac{\left[P\left(\omega_{j}\right) \Delta \omega_{j}\right]^{N_{j}}}{N_{j} !},
\end{aligned}
$$

where $N_{j}$ is the occupation number of the energy $\omega_{j}$,

$$
\mathcal{P}_{\mathrm{el}}(E) \equiv\left|S_{\mathrm{el}}(\boldsymbol{b}=0, s)\right|^{2}=\mathrm{e}^{-2 \pi E R}
$$

is the suppressed elastic scattering probability and

$$
\begin{aligned}
& P(\omega) \equiv \mathrm{e}^{2 \pi \omega R} p(\omega), \\
& p(\omega) \sim \alpha_{G} /\left(\pi \omega^{3} R^{2}\right) \times\left[1-\mathcal{O}\left(\mathrm{e}^{-2 \pi \omega R}\right)\right]
\end{aligned}
$$

is the enhanced single-graviton emission probability density, expressed in terms of $p(\omega)$ which is obtained from the $x$-integration of the leading rescattering term with important subleading corrections for $\omega R \lesssim 1$. In more detail, referring to one jet, we have from Eq. (3.2) — by use of the Parseval identity - the estimate of the rescattering density integrated over the solid angle $\Omega$ for $|\boldsymbol{x}| \gtrsim \mathcal{O}(R) \gg|\boldsymbol{b}|{ }^{4}$

\footnotetext{
${ }^{3}$ Here we treat in detail, for definiteness, the $b \ll R$ case, but our arguments about suppression and compensation are valid for general $b<b_{c}$ by just replacing $\pi$ by $2 \Im \Delta(b)$.

${ }^{4}$ Integrating over $\boldsymbol{q}$ phase space in Eq. (3.6) limits $|\boldsymbol{q}|=\omega \sin \theta<\omega=\mathcal{O}(1 / R)$ and thus introduces a natural cutoff $|\boldsymbol{x}|>R$ in Eq. (3.10) for the validity of the Parseval identity at small $x$-values.
} 


$$
\begin{aligned}
\omega P(\omega)= & \int \sum_{\lambda}\left|\mathfrak{M}_{\lambda}\right|^{2} 2 \omega^{2} \mathrm{~d} \Omega \\
= & \alpha_{G} \int \frac{\mathrm{d}^{2} \boldsymbol{x} 4(2 \pi)^{2}}{(2 \pi|\boldsymbol{x}|)^{4} \omega^{2}}\left|\mathrm{e}^{\omega R[\pi+2 \mathrm{i} \Delta(-\boldsymbol{x})]}-1\right|^{2} \\
\simeq & \frac{\alpha_{G}}{\pi \omega^{2} R^{2}} \mathrm{e}^{2 \pi \omega R}\left[\left(1-\mathrm{e}^{-\pi \omega R}\right)^{2}\right. \\
& \left.+4\left\langle\sin ^{2} \omega R \Delta(\boldsymbol{x})\right\rangle \mathrm{e}^{-\pi \omega R}\right] \\
\equiv & \omega p(\omega) \mathrm{e}^{2 \pi \omega R},
\end{aligned}
$$

where $\langle\cdots\rangle$ denotes $\boldsymbol{x}$-integration (averaging). We thus realize by Eq. (3.7) that the suppression factor $\mathcal{P}_{\mathrm{el}}(E)$ is compensated by the rescattering enhancement factors $\mathrm{e}^{2 \pi \omega_{j} R}$ in $P\left(\omega_{j}\right)$ provided $\sum_{j} \omega_{j} N_{j}=E$, that is close to the energy conservation boundary in which the whole energy $E$ is radiated off. That compensation may occur for a few hard gravitons as well as a bunch of soft ones with $\omega_{j} R=\mathcal{O}(1)$, thus allowing, in principle, a unitary behavior with a normal quasitemperature of order $1 / R$.

\section{UNITARITY RESTORATION AND QUASITEMPERATURE: THE RESCATTERING TERMS}

In order to better understand how suppression is avoided and unitarity is possibly restored in the rescattering case, note that the expressions (3.7) and (3.10) allow the use of the energy-conserving unitarization method based on the $\mathcal{N}(E)$ rescaling $[20,29,30]$ in the $b<b_{c}$ case also. In fact we can replace the independent-particle distribution (3.7) by

$$
\begin{aligned}
\mathrm{d} \tilde{\mathcal{P}}\left(\left\{\omega_{j}, N_{j}\right\}\right)= & \frac{\mathcal{P}_{\mathrm{el}}(E)}{\mathcal{N}(E)} \int_{c-\mathrm{i} \infty}^{c+\mathrm{i} \infty} \frac{\mathrm{d} \lambda}{2 \pi \mathrm{i} \lambda} \mathrm{e}^{\lambda\left(E-\sum_{j} \omega_{j} N_{j}\right)} \\
& \times \prod_{j} \frac{\left[P\left(\omega_{j}\right) \Delta \omega_{j}\right]^{N_{j}}}{N_{j} !}
\end{aligned}
$$

where $\mathcal{P}_{\text {el }} / \mathcal{N}$ plays a role similar to the $b>b_{c}$ case, but $\mathcal{P}_{\mathrm{el}}=\mathrm{e}^{-2 \pi E R}$ is the ACV-resummed suppression result. What we are really assuming here is that the multigraviton emission amplitudes (including higher order terms playing the role of virtual corrections) keep the eikonal factorization structure (3.3), apart from the positive rescaling factor $1 / \sqrt{\mathcal{N}(E)}$, even in the case in which the elastic channel carries a suppression factor and the emission ones various enhancement factors.

Then, the unitarity requirement $\sum_{\left\{N_{j}\right\}} \mathrm{d} \tilde{\mathcal{P}}\left(\left\{\omega_{j}, N_{j}\right\}\right)=1$ (with the kinematical constraint $\sum_{j} \omega_{j} N_{j} \leq E$ ) determines $\mathcal{N}(E)$ as

$$
\begin{aligned}
\mathcal{N}(E) \equiv & \mathcal{P}_{\mathrm{el}}(E)\left[1+\sum_{N=1}^{\infty} \frac{1}{N !} \int\left(\prod_{j=1}^{N} \mathrm{~d} \omega_{j} P\left(\omega_{j}\right)\right)\right. \\
& \left.\Theta\left(E-\sum_{j=1}^{N} \omega_{j}\right)\right] \\
= & \int_{2 \pi R-\mathrm{i} \infty}^{2 \pi R+\mathrm{i} \infty} \frac{\mathrm{d} \lambda}{2 \pi \mathrm{i} \lambda} \mathcal{P}_{\mathrm{el}}(E) \mathrm{e}^{\lambda E+\int_{0}^{\infty} \mathrm{d} \omega \mathrm{e}^{-\lambda \omega} P(\omega)},
\end{aligned}
$$

where we have set $\mathfrak{i} \lambda \geq c=2 \pi R$ to let the exponent integrand formally converge. By the translation $\lambda=$ $(2 \pi+\tau) R$ we then obtain

$$
\mathcal{N}(E)=\int_{-\mathrm{i} \infty}^{+\mathrm{i} \infty} \frac{\mathrm{d} \tau}{2 \pi \mathrm{i}(\tau+2 \pi)} \mathrm{e}^{\tau R E+\int_{0}^{\infty} \mathrm{d} \omega \mathrm{e}^{-\tau \omega R} p(\omega)},
$$

where $\mathcal{P}_{\mathrm{el}}$ has been replaced by 1 and $P(\omega)$ by $p(\omega)$ of Eq. (3.10). ${ }^{5}$ Therefore, all suppression and enhancement factors are now eliminated and the determination of the energy-conserving $\mathrm{d} \tilde{\mathcal{P}}$ 's can proceed as for $b>b_{c}$.

First of all, the distribution (4.1) in the $\tau$-representation becomes

$$
\begin{aligned}
\mathrm{d} \tilde{\mathcal{P}}= & \frac{1}{\mathcal{N}(E)} \int_{\epsilon-\mathrm{i} \infty}^{\epsilon+\mathrm{i} \infty} \frac{\mathrm{d} \tau}{2 \pi \mathrm{i}(\tau+2 \pi)} \mathrm{e}^{\tau R\left(E-\sum_{j} \omega_{j} N_{j}\right)} \\
& \times \prod_{j} \frac{\left[p\left(\omega_{j}\right) \Delta \omega_{j}\right]^{N_{j}}}{N_{j} !}
\end{aligned}
$$

where $\mathcal{N}(E)$ in (4.2) essentially exponentiates the single-emission graviton multiplicity (of order $\alpha_{G}$ ), subject to the kinematical constraints and to the suppression and enhancement factors. Its estimate can be given in the $\tau$-representation (4.3) by a saddle point method around some $\tau=\bar{\tau}>0$. For instance, in the toy example $p(\omega)=\alpha_{G} R \hat{p}=$ const, we find directly $\bar{\tau}=\sqrt{\hat{p}}$ and, for $E=\sqrt{s} / 2-\omega, \quad \mathcal{N}(E) \sim \exp \left[2 \sqrt{\alpha_{G} E R \hat{p}}\right] \sim$ $\exp \left[2 \alpha_{G} \bar{\tau} \sqrt{1 \frac{\omega}{\sqrt{s} / 2}}\right]$ illustrating the fact that $\mathcal{N}(E) \gg 1$ despite the suppression factor $\mathcal{P}_{\mathrm{el}}(E)$ in Eq. (4.2). That suggests the tentative interpretation of $\mathcal{N}(E)$ as the effective number of states available to the trapped energy $E$ fragmentation process. Correspondingly, $1 / \mathcal{N}(E)$ is the analogue, for $b<b_{c}$, of the no-emission probability in Eq. (3.4) which, for $b>b_{c}$, plays the role of virtual corrections.

\footnotetext{
${ }^{5}$ In writing (4.2) [(4.3)] we have to exchange the order of $\lambda(\tau)$-integrations with $\omega$-integrations. That is simply achieved by the truncation of $P(\omega)$ at the kinematical boundary: $P(\omega) \rightarrow P(\omega) \Theta(\sqrt{s} / 2-\omega)$. That truncation is understood in the following, and is a consequence of the kinematical constraints also for $E \leq \sqrt{s} / 2$. We note that $E$ in Eq. (4.4) varies from the "on-shell" value $E=\sqrt{s} / 2$ depending on the type of measurement being made, $E=\sqrt{s} / 2-\sum_{j} \omega_{j} N_{j}$ if associated with the emitted graviton energies $\omega_{j}$.
} 
Secondly, the general saddle point equation reads

$$
\begin{aligned}
\alpha_{G}= & \frac{1}{\bar{\tau}+2 \pi}+\alpha_{G} F(\bar{\tau}) \\
F(\bar{\tau}) \equiv & \int_{0}^{\infty} \mathrm{d} \omega \frac{\omega}{E} \mathrm{e}^{-\tau \omega R} p(\omega) \\
= & \int_{0}^{\infty} \mathrm{d}(\omega R) \frac{\alpha_{G}}{\pi(\omega R)^{2}}\left[\left(1-\mathrm{e}^{-\pi \omega R}\right)^{2}\right. \\
& \left.+4\left\langle\sin ^{2} \omega R \Delta(\boldsymbol{x})\right\rangle \mathrm{e}^{-\pi \omega R}\right] \mathrm{e}^{-\bar{\tau} \omega R},
\end{aligned}
$$

which for $\alpha_{G} \gg 1$ leads to approximately $F(\bar{\tau})=1$, that is to $\sum_{j} \omega_{j} N_{j}=E$, or radiation of the total trapped energy, as suggested by Eq. (3.7).

Furthermore, we can calculate from Eq. (4.1) the singlegraviton inclusive distribution $\mathrm{d} N / \mathrm{d} \omega$, which involves fixing $\omega_{j}=\omega$ for some $j$, and integrating over the remaining ones at $\sqrt{s} / 2-\omega$ fixed. It is straightforward to see that this provides, apart from small fluctuation corrections $\mathcal{O}\left(1 / \alpha_{G}\right)$,

$$
\frac{\mathrm{d} N}{\mathrm{~d} \omega}=p(\omega) \frac{\mathcal{N}(\sqrt{s} / 2-\omega)}{\mathcal{N}(\sqrt{s} / 2)} \simeq p(\omega) \mathrm{e}^{-\bar{\tau} R \omega},
$$

which is consistent with the average emitted energy in (4.5) and, together with Eqs. (4.3)-(4.5), appears to solve the $b \ll R$ model with a meaningful unitarization.

The exponential damping at large $\omega R$, which affects all the inclusive distributions as in Eq. (4.6), allows us to interpret $(\bar{\tau} R)^{-1}$ as the quasitemperature of graviton radiation, and provides an effective cutoff in frequency, superimposed to the density $p(\omega)$, typical of our soft-based representation. The overall picture of the "trapped-energy" fragmenting into soft gravitons according to the distribution (4.4) looks generically compatible with ideas discussed in Refs. [21,22], although applied - in our case - to the precise ACV framework at fixed impact parameter $b$ and with $s$-channel iteration. Arguments for a cutoff are given also in the approach of Ref. [22] to the trans-Planckian scattering without impact parameter identification of Ref. [21].

The precise determination of the inverse quasitemperature $\bar{\tau}$ for rescattering requires a small- $x$ cutoff parameter $|\boldsymbol{x}|_{\text {cut }}=\mathcal{O}(R)$ which takes contributions from $\Im \Delta(\boldsymbol{x})>0$ if $|\boldsymbol{x}|_{\text {cut }}^{2}<b_{c}^{2}=(3 \sqrt{3} / 2) R^{2}$, a region where the $\boldsymbol{x}$-dependence starts being suppressed in (4.5). We then find the numerical results of Fig. 3, showing that $\bar{\tau} \simeq 3$ is a reasonable estimate (solid-blue curve), to be compared with $\bar{\tau}=1.2$ from $b \rightarrow b_{c}^{+}$[20]. We should notice, however, that $\bar{\tau}$ is rather sensitive to the value of $|\boldsymbol{x}|_{\text {cut }}$ around and below $b_{c}$. That means that the inclusion of incidencechanging contributions to radiation may possibly be needed to provide a more stable temperature estimate and a firmer conclusion on the unitary behavior on the basis of Eqs. (4.3) and (4.5).

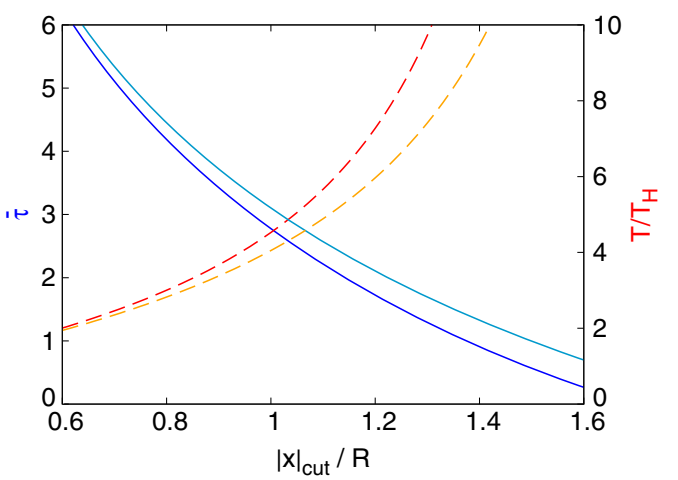

FIG. 3. Dependence of the saddle point value $\bar{\tau}$ (solid blue, left axis) and of the rescattering quasitemperature $T \equiv 1 /(\bar{\tau} R)$ (dashed red, right axis) in units of $T_{H} \equiv 1 /(4 \pi R)$ on the cutoff parameter $|\boldsymbol{x}|_{\text {cut }}$ introduced in the approximate determination of the saddle point Eq. (4.5). Including incidence-changing effects at first order (see Sec. V) the saddle point values increase (solid light blue) and correspondingly the quasitemperature decreases (dashed orange).

We should add finally that, starting from Eq. (3.1), we can also compute the probability distribution for the residual energy of the trapped gravitons $E^{\prime}=$ $E-\sum_{j} \omega_{j} N_{j}$. By introducing the $\delta$-function constraint, we get the formula

$$
\begin{aligned}
\frac{\mathrm{d} \mathcal{P}}{\mathrm{d} E^{\prime}} & =\frac{\mathcal{P}_{\mathrm{el}}(E)}{\mathcal{N}(E)} \sum_{\left\{N_{j}\right\}} \delta\left(E-E^{\prime}-\sum_{j} \omega_{j} N_{j}\right) \prod_{j} \frac{\left[P\left(\omega_{j}\right) \Delta \omega_{j}\right]^{N_{j}}}{N_{j} !} \\
& =\frac{\Theta\left(E^{\prime}\right)}{\mathcal{N}(E)} \mathrm{e}^{-2 \pi R E^{\prime}} \int_{-\mathrm{i} \infty}^{+\mathrm{i} \infty} \frac{\mathrm{d} \tau^{\prime} R}{2 \pi \mathrm{i}} \mathrm{e}^{\tau^{\prime}\left(E-E^{\prime}\right) R+\int_{0}^{\infty} \mathrm{d} \omega p(\omega) \mathrm{e}^{-\omega R \tau^{\prime}}},
\end{aligned}
$$

which can be estimated by a saddle point method also.

We note that the unitarity condition $\int_{0}^{\infty} \mathrm{d} E^{\prime} \mathrm{d} \mathcal{P} / \mathrm{d} E^{\prime}=1$ fixes again $\mathcal{N}(E)$ as in Eq. (4.3) by reproducing the translation $\tau^{\prime} \rightarrow \tau^{\prime}+2 \pi$ in the denominator. Indeed, the $E^{\prime}$ distribution is mostly dependent on the tunneling exponent $2 \pi R$, which appeared in $\mathcal{P}_{\text {el }}(E)$. At $E^{\prime}$ fixed the saddle point $\bar{\tau}^{\prime}$ is determined by

$$
F\left(\bar{\tau}^{\prime}\right)=1-\frac{E^{\prime}}{E}\left(\simeq 1-\frac{1}{\alpha_{G}(\bar{\tau}+2 \pi)}\right)
$$

and the average $\left\langle E^{\prime}\right\rangle \simeq 1 /(2 \pi R+\bar{\tau} R)$ makes $\bar{\tau}^{\prime}$ roughly consistent with $\bar{\tau}$ in Eq. (4.5), with a maximal residual "bound" energy of order $1 /(2 \pi R)$. It seems therefore that both the "bulk" temperature $1 /(\bar{\tau} R)$ and the residual-energy temperature $1 /(2 \pi R)$ (reminiscent of the Schwarzschild black-hole limit [12]) play a role in this model. We stress the point, however, that, because of (4.7), they are consistent with each other, due to their different definition. 


\section{INCIDENCE-CHANGING CONTRIBUTIONS}

We have so far considered the rescattering terms in (3.1) and (3.2), because they offer the first exit window at $|x| \gtrsim R$. Now we want to estimate the incidence-changing window at varying values of $\frac{\omega}{E}|\boldsymbol{x}|$, in which the parameter $\omega / E$ affects directly the $\boldsymbol{x}$-dependence.

The single-emission density of Eq. (3.10) becomes, approximately,

$\omega P(\omega) \simeq \frac{\alpha_{G}}{\pi \omega^{2}\left\langle\boldsymbol{x}^{2}\right\rangle}\left|\mathrm{e}^{\omega R[\pi+2 \mathrm{i} \Delta(-\boldsymbol{x})]}-\mathrm{e}^{\alpha_{G}\left[\pi+2 \mathrm{i} \Delta\left(-\frac{\omega}{E} x\right)\right]}\right|^{2}$,

while the elastic scattering probability is $\mathcal{P}_{\text {el }}=\mathrm{e}^{-2 \pi \alpha_{G}}$ as usual for $b \ll R$. We note that in the incidencechanging term the enhancement occurs at coupling $\alpha_{G}>\omega R$, but there is further suppression, due to the $\omega / E$ dependence in the eikonal expansions at small $\omega|\boldsymbol{x}|$ values $\left(\frac{b}{R} \ll \frac{\omega|x|}{\mathrm{ER}} \ll 1\right)[12]$

$2 \Delta\left(-\frac{\omega}{E} x\right) \simeq \mathrm{i} \pi-3 \mathrm{e}^{\mathrm{i} \pi / 3}\left(\frac{\omega|x|}{\mathrm{ER}}\right)^{2 / 3}+\mathcal{O}\left(\frac{b^{2}}{R^{2}}\right)^{1 / 3}$,

$$
\begin{aligned}
& \mathrm{e}^{\alpha_{G}\left[\pi+2 \mathrm{i} \Delta\left(-\frac{\omega}{E} x\right)\right]} \simeq \mathrm{e}^{a \alpha_{G}\left(\frac{\omega R}{\alpha_{G}}\right)^{2 / 3}}, \\
& \left(a=\frac{3 \sqrt{3}}{2} \text { for }\left\langle x^{2}\right\rangle=R^{2}\right) .
\end{aligned}
$$

We thus realize, by (5.1) and (5.3), that we should consider two regions for incidence-changing terms. In the region $|x| \geq \frac{E}{\omega} R$ the trapping suppression is indeed canceled out, but the outcome is reduced by the phase space factor $(\omega / E)^{2}$, yielding small $\left(\sim 1 / \alpha_{G}\right)$ contributions in the hard-graviton corner. On the other hand, in the region $|x| \gtrsim R$ we are interested in, there is a small$\omega R$ configuration $\left(\omega R / \alpha_{G}\right)^{1 / 3}<a / \pi<1$ in which the enhancement (5.3), though insufficient by itself to overcome the damping, is anyway larger than the single-density rescattering contribution, and therefore should be taken into account. To this purpose, we can provide from (5.3) a crude estimate of the Laplace transform

$\tilde{P}_{\text {ic }}(\lambda) \equiv \int_{0}^{\alpha_{G}} \mathrm{~d} \omega P_{\text {ic }}(\omega) \mathrm{e}^{-\omega \lambda} \simeq\left\{\begin{array}{ll}\mathrm{e}^{\frac{2}{3} a \alpha_{G}\left(\frac{4 a}{3 \lambda}\right)^{2}} & \left(\frac{4 a}{3 \lambda}<1\right) \\ \mathrm{e}^{(2 a-\lambda) \alpha_{G}} & \left(\frac{4 a}{3 \lambda} \geq 1\right)\end{array}\right.$,

where the last expression is provided by a saddle point at $\bar{\omega} R=\alpha_{G}\left(\frac{4 a}{3 \lambda}\right)^{3}$, if below $\bar{\omega} R=\alpha_{G}$, and by the end point $\omega R=\alpha_{G}$ otherwise. We note that

$$
-\frac{\tilde{P}_{\mathrm{ic}}^{\prime}(\lambda)}{\tilde{P}_{\mathrm{ic}}(\lambda)}=\left\{\begin{array}{ll}
\alpha_{G}\left(\frac{4 a}{3 \lambda}\right)^{3}=\bar{\omega} R & \left(\frac{4 a}{3 \lambda}<1\right) \\
\alpha_{G} & \left(\frac{4 a}{3 \lambda} \geq 1\right)
\end{array} .\right.
$$

Our task, however, should be to provide a reliable estimate of the incidence-changing terms at the manygraviton level required by Eq. (4.4), and that raises a variety of questions, involving both matter of concept (factorization is justified for rescattering terms only) and technical approximations for $b \ll R$. Therefore, further analysis is needed and treating incidence-changing contributions in detail in addition to rescattering ones is outside the scope of the present paper.

We only point out that the rescattering estimate of $\mathcal{N}(E)$ in the $\tau$-representation (4.3) can be improved by including incidence-changing effects at the level of single power of $P_{\text {ic }}(\omega)$. In that case the exponent in the integrand of (4.3) is corrected by adding a $\log \tilde{p}_{\text {ic }}(2 \pi+\tau)$ from (5.4) and the saddle point equation (4.5) becomes

$$
\alpha_{G}=\frac{1}{\bar{\tau}+2 \pi}+\alpha_{G}\left[F(\bar{\tau})+\left(\frac{4 a}{3(\bar{\tau}+2 \pi)}\right)^{3}\right],
$$

where the logarithmic derivative is taken from (5.5). The meaning of (5.6) is that a fraction of the overall energy is now radiated by $P_{\text {ic }}$ also. The addition of such an incidence-changing effect on the saddle point and temperature values is shown in Fig. 3. There is a moderate increase in $\bar{\tau}$ (solid-light-blue curve) and a corresponding decrease in temperature (dashed-orange curve).

\section{DISCUSSION}

Here we have investigated the collapse regime of gravitational scattering at extreme energies $\left(E \gg M_{p}\right)$ and small impact parameters $\left(b<b_{c} \sim R\right)$, and we have pointed out that - in the present approach — graviton radiation is not necessarily suppressed, and actually multigraviton amplitudes suggest how a unitary $S$-matrix may still be found.

Our framework is semiclassical scattering [7] in the ACV-resummed [12] eikonal formulation and in the soft-based representation [20] of graviton radiation. Our suggestion is based on two main points. First, starting from jet energy $E=\sqrt{s} / 2$ at impact parameter $b \ll R \equiv$ $4 G E$ elastic scattering to free-particle states is exponentially suppressed by $\left|S_{\mathrm{el}}(b)\right| \simeq \mathrm{e}^{-\pi E R}$ because of $\Im \Delta(b) \simeq$ $\pi / 2$ [Eq. (1.4)]. But rescattering contributions of an emitted graviton at position $\boldsymbol{x}$ are regulated by $\Delta(\boldsymbol{b}-\boldsymbol{x})$ [Eq. (3.1)] and are thus unsuppressed if $|\boldsymbol{x}|^{2}>b_{c}^{2} \equiv$ $(3 \sqrt{3} / 2) R^{2} \gg b^{2}$. That large-distance radiation is in turn associated to the recoil energy $E-\omega$ of the energetic particle, so that the suppression amplitude is reduced-and the emission one is enhanced-by the factor $\mathrm{e}^{\pi \omega R}$, within 
the kinematical bounds. The same conclusion is reached for general $b<b_{c}$ by replacing $\pi$ by $2 \Im \Delta(b)$.

Secondly, that suppression-enhancement correspondence goes through to multigraviton states provided the related soft-graviton $\left(\omega_{j} \ll E\right)$ amplitudes are factorized, as argued for in [20] on the basis of eikonal factorization, at least for rescattering amplitudes. In such a case, the overall suppression factor in multigraviton emission becomes

$$
\exp \left[-\pi\left(E-\sum_{j=1}^{N} \omega_{j}\right) R\right] \Theta\left(E-\sum_{j=1}^{N} \omega_{j}\right)
$$

and is therefore $\mathcal{O}(1)$ (meaning no suppression) if $E=$ $\sum_{j} \omega_{j}$ or, in other words, if the whole energy is radiated off. That may happen for a few hard gravitons, but also for a bunch of soft ones, thus allowing, in principle, unsuppressed emission amplitudes with a normal quasitemperature of order $1 / R$.

Therefore, if we take multigraviton factorization for granted, the above argument hints at the probability distribution (4.4), which is unitarized by the normalization factor $\mathcal{N}(E)$ in (4.3) and is characterized by the saddle point $\bar{\tau}$ in (4.5), the inclusive distribution (4.6) and thus the quasitemperature $1 /(\bar{\tau} R)$.

This looks to be the right path to follow in general, but unfortunately we do not quite understand how to combine the incidence-changing contributions with the rescattering ones at the multigraviton level required by (6.1), because of their uncertain factorization properties. That is not surprising, because the incidence-changing terms (which regulate the rotation of the incidence axis) are basically dependent on the overall coupling $\alpha_{G}$ of the energetic particles, while their $\omega_{j} R$-dependence is tied up with the $\boldsymbol{x}$-dependence and is normally nonfactorizable.

For those reasons, in Sec. IV we concentrate on the rescattering terms (for which multigraviton factorization is justified) by introducing a cutoff $|\boldsymbol{x}|_{\text {cut }}=\mathcal{O}(R)$, to regulate small- $x$ contributions. We thus find that the unitarization method suggested by (6.1) actually works, with some cutoff dependence of $\bar{\tau}$ with $\bar{\tau} \simeq 3$ for $|x|_{\text {cut }}=R$.
We feel that a better understanding of incidence-changing contributions could be able to close the gaps and reduce the cutoff dependence, as suggested by the provisional estimate of Fig. 3. Such an analysis is deferred to further investigations.

A perhaps more fundamental question to be discussed is what our results in Sec. IV actually mean for the gravitational scattering and, possibly, for black-hole physics. We have already noticed that there are two ways the trapped energy $\sqrt{s}=2 E$ can be observed: either in full, without accompanying soft gravitons, by the amplitude $\sim \mathrm{e}^{-\pi \alpha_{G}}$ [corresponding to the residual energy temperature $1 /(2 \pi R)]$ which is exponentially suppressed, or instead by a sort of collective fragmentation into soft gravitons, described by the distribution (4.4) and the quasitemperature $1 /(\bar{\tau} R)$. Shall we conclude that the latter is the most probable issue and that, therefore, the unitary distribution (4.4) represents the quantum black-hole spectrum?

If that is really the case, then the solution of the unitarity problem and, perhaps, of the information paradox in blackhole fragmentation would rely only on our ability to keep track of the phases and to describe the quantum states. That brings us back to the previous question of whether or not we are able to disentangle the full multigraviton amplitudes, and that, finally, seems to be a matter of technique and not a matter of principle.

To conclude, we are aware of the fact that our framework is not a consistent quantum gravity theory and is thus providing a limited description of gravitational processes. Nevertheless, our discussion suggests that some difficulties previously found with unitarity and the information paradox may be solved by our proposal in a simpler way than previously thought.

\section{ACKNOWLEDGMENTS}

It is a pleasure to thank Gabriele Veneziano for a number of interesting conversations on the topics presented in this paper. We also thank the Galileo Galilei Institute for Theoretical Physics for hospitality while part of this work was being done.
[1] G. 't Hooft, Phys. Lett. B 198, 61 (1987).

[2] I. J. Muzinich and M. Soldate, Phys. Rev. D 37, 359 (1988).

[3] D. Amati, M. Ciafaloni, and G. Veneziano, Phys. Lett. B 197, 81 (1987).

[4] D. Amati, M. Ciafaloni, and G. Veneziano, Int. J. Mod. Phys. A 03, 1615 (1988).

[5] H. L. Verlinde and E. P. Verlinde, Nucl. Phys. B371, 246 (1992).
[6] D. Amati, M. Ciafaloni, and G. Veneziano, Nucl. Phys. B347, 550 (1990).

[7] D. Amati, M. Ciafaloni, and G. Veneziano, Nucl. Phys. B403, 707 (1993).

[8] M. Ciafaloni and D. Colferai, J. High Energy Phys. 10 (2014) 85.

[9] X. O. Camanho, J. D. Edelstein, J. Maldacena, and A. Zhiboedov, J. High Energy Phys. 02 (2016) 020. 
[10] G. D’Appollonio, P. Di Vecchia, R. Russo, and G. Veneziano, J. High Energy Phys. 05 (2015) 144.

[11] L. Lipatov, Sov. Phys. JETP 55, 582 (1982).

[12] D. Amati, M. Ciafaloni, and G. Veneziano, J. High Energy Phys. 02 (2008) 049.

[13] G. Marchesini and E. Onofri, J. High Energy Phys. 06 (2008) 104.

[14] G. Veneziano and J. Wosiek, J. High Energy Phys. 09 (2008) 023.

[15] G. Veneziano and J. Wosiek, J. High Energy Phys. 09 (2008) 024.

[16] M. Ciafaloni and D. Colferai, J. High Energy Phys. 11 (2008) 047.

[17] A. Gruzinov and G. Veneziano, Classical Quantum Gravity 33, 125012 (2016).

[18] M. Ciafaloni, D. Colferai, and G. Veneziano, Phys. Rev. Lett. 115, 171301 (2015).

[19] M. Ciafaloni, D. Colferai, F. Coradeschi, and G. Veneziano, Phys. Rev. D 93, 044052 (2016).
[20] M. Ciafaloni and D. Colferai, Phys. Rev. D 95, 086003 (2017).

[21] G. Dvali, C. Gomez, R. Isermann, D. Lüst, and S. Stieberger, Nucl. Phys. B893, 187 (2015).

[22] A. Addazi, M. Bianchi, and G. Veneziano, J. High Energy Phys. 02 (2017) 111.

[23] S. Weinberg, Phys. Rev. 140, B516 (1965).

[24] S. W. Hawking, Commun. Math. Phys. 43, 199 (1975).

[25] S. W. Hawking, arXiv:1509.01147.

[26] M. Ciafaloni and D. Colferai, J. High Energy Phys. 12 (2009) 062.

[27] M. Ciafaloni, D. Colferai, and G. Falcioni, J. High Energy Phys. 09 (2011) 044.

[28] L. N. Lipatov, Nucl. Phys. B365, 614 (1991).

[29] H. Grigoryan and G. Veneziano (private communication).

[30] V. A. Abramovsky, V. N. Gribov, and O. V. Kancheli, Yad. Fiz. 18, 595 (1973) [Sov. J. Nucl. Phys. 18, 308 (1974)]. 